\title{
Monte Carlo analysis of MEGA microlensing events towards M 31
}

\author{
G. Ingrosso ${ }^{1}$, S. Calchi Novati ${ }^{2}$, F. De Paolis ${ }^{1}$, Ph. Jetzer $^{2}$, A. A. Nucita ${ }^{1}$, and F. Strafella ${ }^{1}$ \\ 1 Dipartimento di Fisica, Università di Lecce and INFN, Sezione di Lecce, CP 193, 73100 Lecce, Italy \\ 2 Institute for Theoretical Physics, University of Zürich, Winterthurerstrasse 190, 8057 Zürich, Switzerland
}

Received 24 May 2005 / Accepted 6 September 2005

\begin{abstract}
We perform an analytical study and a Monte Carlo (MC) analysis of the main features for microlensing events in pixel lensing observations towards M31. Our main aim is to investigate the lens nature and location of the 14 candidate events found by the MEGA collaboration. Assuming a reference model for the mass distribution in M31 and the standard model for our galaxy, we estimate the MACHO-to-self lensing probability and the event time duration towards M31. Reproducing the MEGA observing conditions, as a result we get the MC event number density distribution as a function of the event full-width half-maximum duration $t_{1 / 2}$ and the magnitude at maximum $R_{\max }$. For a MACHO mass of $0.5 M_{\odot}$ we find typical values of $t_{1 / 2} \simeq 20$ day and $R_{\max } \simeq 22$, for both MACHO-lensing and self-lensing events occurring beyond about 10 arcmin from the M31 center. A comparison of the observed features $\left(t_{1 / 2}\right.$ and $\left.R_{\max }\right)$ with our MC results shows that for a MACHO mass $>0.1 M_{\odot}$ the four innermost MEGA events are most likely self-lensing events, whereas the six outermost events must be genuine MACHO-lensing events.
\end{abstract}

Key words. gravitational lensing - galaxy: halo - galaxies: individuals: M31

\section{Introduction}

Gravitational microlensing has become since about a decade a robust tool for analyzing the galactic structure and for gaining information about the dark mass component in our galaxy (Alcock et al. 1993; Aubourg et al. 1993). Several hundreds of microlensing events have been detected so far towards the galactic bulge, the spiral arms, and the Magellanic Clouds (Alcock et al. 2000; Jetzer et al. 2004).

Recently, pixel lensing observations towards M 31 and even M 87 have been undertaken and some microlensing events have been found. The MEGA collaboration (de Jong et al. 2004) has reported the detection of 14 candidate events towards M 31 by using Isaac Newton Telescope (INT) data. Two of these events have been previously reported by the POINT-AGAPE collaboration (Paulin-Henriksson et al. 2003), which recently has presented a high-threshold analysis of the full 3 years data set (Calchi Novati et al. 2005). This analysis shows that the observed signal is much larger than expect from self-lensing alone and that some fraction of the halo mass must be in form of MACHOs. Other collaborations have also reported preliminary results for pixel lensing towards M 31 (Calchi Novati et al. 2002, 2003; Uglesich et al. 2004; Riffeser et al. 2003; Joshi et al. 2004).

In this paper we consider in particular the MEGA results which provide the largest sample of microlensing candidates. A map of the MEGA events is reported by de Jong et al. (2004). A preliminary analysis by the MEGA collaboration indicates that the events located in the outer part of M31 are consistent with being due to halo lens objects, whereas the innermost ones are most likely due to self-lensing. The aim of this paper is to perform a more complete analysis by using a Monte Carlo (MC) program with the purpose of using all available information by the MEGA collaboration, which are summarized in Table 1, to characterize the nature of the lenses.

The paper is organized as follows: in Sect. 2 we present the calculation of the microlensing rate towards M 31 and we remind the pixel lensing basics. In Sect. 3 we give some details on the adopted M 31 and Milky Way (MW) mass distribution models as well as the stellar and mass functions. The analytic and $\mathrm{MC}$ results and a comparison between the two are given in Sects. 4-6 and conclusions are presented in Sect. 7.

\section{Microlensing rate}

The differential number of expected microlensing events is (De Rújula et al. 1991; Griest 1991)

$\mathrm{d} N_{\mathrm{ev}}=N_{*} t_{\mathrm{obs}} \mathrm{d} \Gamma$,

where $N_{*}$ is the total number of monitored stars during the observation time $t_{\mathrm{obs}}$. The differential rate $\mathrm{d} \Gamma$ at which a single star is microlensed by a compact object is given by

$\mathrm{d} \Gamma=\frac{n_{1}(\boldsymbol{x}, \mu) \mathrm{d} \mu f\left(\boldsymbol{v}_{1 \perp}\right) \mathrm{d}^{2} \boldsymbol{v}_{1 \perp} \mathrm{d}^{3} x}{\mathrm{~d} t}$,

where the numerator on the right hand side is the number of lenses with transverse velocity in $\mathrm{d}^{2} \boldsymbol{v}_{1 \perp}=v_{1 \perp} \mathrm{d} v_{1 \perp} \mathrm{d} \beta$ 
Table 1. For the 14 MEGA events we give the position, the full-width half-maximum duration $t_{1 / 2}$ and the magnitude at maximum $R_{\max }$. The coordinate system we adopt has origin in the M31 center and the $\mathrm{X}$ axis is oriented along the M 31 disk major axis (see also Fig. 1).

\begin{tabular}{ccccc}
\hline \hline MEGA & $\begin{array}{c}X \\
\operatorname{arcmin}\end{array}$ & $\begin{array}{c}Y \\
\operatorname{arcmin}\end{array}$ & $\begin{array}{c}t_{1 / 2}^{\mathrm{obs}} \\
\text { day }\end{array}$ & $\begin{array}{c}R_{\max }^{\mathrm{obs}} \\
\operatorname{magn}\end{array}$ \\
\hline 1 & -4.367 & -2.814 & $4.20 \pm 4.30$ & $22.2 \pm 1.1$ \\
2 & -4.478 & -3.065 & $4.60 \pm 0.60$ & $21.6 \pm 0.3$ \\
3 & -7.379 & -1.659 & $2.60 \pm 2.20$ & $21.8 \pm 1.2$ \\
4 & -10.219 & 3.420 & $29.10 \pm 1.00$ & $22.8 \pm 0.2$ \\
5 & -19.989 & -13.955 & $9.40 \pm 4.10$ & $22.9 \pm 0.8$ \\
6 & -21.564 & -13.169 & $22.90 \pm 0.70$ & $22.6 \pm 0.2$ \\
7 & -21.163 & -6.230 & $21.60 \pm 0.70$ & $19.3 \pm 0.2$ \\
8 & -21.650 & 7.670 & $27.40 \pm 0.90$ & $22.7 \pm 0.2$ \\
9 & -33.834 & -2.251 & $3.80 \pm 1.60$ & $21.8 \pm 0.8$ \\
10 & -3.933 & -13.846 & $46.80 \pm 4.40$ & $22.2 \pm 0.3$ \\
11 & 19.192 & -11.833 & $2.00 \pm 0.30$ & $20.5 \pm 0.2$ \\
12 & 29.781 & -5.033 & $131.00 \pm 9.40$ & $23.2 \pm 0.3$ \\
13 & 22.072 & -22.022 & $22.80 \pm 3.80$ & $23.3 \pm 0.3$ \\
14 & 19.348 & -29.560 & $28.10 \pm 1.40$ & $22.5 \pm 0.2$ \\
\hline
\end{tabular}

around $\boldsymbol{v}_{1 \perp}$, located in a volume element $\mathrm{d}^{3} x=\mathrm{d} x \mathrm{~d} y \mathrm{~d} z$ centered at the position $\boldsymbol{x}$ of the microlensing tube. Here $\boldsymbol{v}_{\perp \perp}$ is the component (in the rest frame of the Galaxy) of the lens velocity orthogonal to the line of sight to the source star, $n_{1}(\boldsymbol{x}, \mu)$ the lens number density (per unit of volume and mass) and $f\left(\boldsymbol{v}_{\perp \perp}\right)$ the lens tranverse velocity distribution. $\mu$ is the lens mass in solar units.

In evaluating $\mathrm{d} \Gamma$ we must take into account that the source stars are not at rest but have a transverse velocity $\boldsymbol{v}_{\mathrm{S} \perp}$ as well, which can be splitted into a random component and a component which describes the ordered rotation (if present) of the galactic component considered, namely $\boldsymbol{v}_{\mathrm{s} \perp}=\boldsymbol{v}_{\mathrm{s} \perp \text {,ran }}+\boldsymbol{v}_{\mathrm{s} \perp \text {,rot }}$. Using a Maxwellian distribution with 2-dimensional velocity dispersion $\sigma_{\mathrm{s}}$ to describe the random velocity component, we get for the transverse velocity distribution $g\left(v_{\mathrm{s} \perp}\right)$

$g\left(\boldsymbol{v}_{\mathrm{S} \perp}\right) v_{\mathrm{S} \perp} \mathrm{d} v_{\mathrm{S} \perp} \mathrm{d} \varphi=\frac{1}{\pi \sigma_{\mathrm{S}}^{2}} \mathrm{e}^{-\frac{\left(v_{\mathrm{s} \perp}-v_{\mathrm{s} \perp \mathrm{r}, \mathrm{rot}}\right)^{2}}{\sigma_{\mathrm{S}}^{2}}} v_{\mathrm{S} \perp} \mathrm{d} v_{\mathrm{S} \perp} \mathrm{d} \varphi$

where $\varphi$ is the angle between $\boldsymbol{v}_{\mathrm{s} \perp}$ and $\boldsymbol{v}_{\mathrm{S} \perp \text {,rot }}$.

We take also into account the transverse rotation velocity of the observer, $\boldsymbol{v}_{\odot \perp \text {,rot }}$ (here we consider the observer co-moving with the Sun) by considering that the microlensing tube is moving with a transverse velocity

$\boldsymbol{v}_{\mathrm{t} \perp}=\left(1-\frac{D_{\mathrm{ol}}}{D_{\mathrm{os}}}\right) \boldsymbol{v}_{\odot \perp, \mathrm{rot}}+\frac{D_{\mathrm{ol}}}{D_{\mathrm{os}}} \boldsymbol{v}_{\mathrm{s} \perp}$

where $D_{\mathrm{os}}$ and $D_{\mathrm{ol}}$ are the source and lens distances from the observer, respectively.

As for the source stars we split the lens transverse velocity into a random component and an ordered rotation component. Moreover, by taking into account that the microlensing tube moves with velocity $\boldsymbol{v}_{\mathrm{t} \perp}$, it follows that $\boldsymbol{v}_{1 \perp}=\boldsymbol{v}_{1 \perp \text {,ran }}+\boldsymbol{v}_{1 \perp \text {,rot }}-$ $\boldsymbol{v}_{\mathrm{t} \perp}$. Accordingly, assuming for $\boldsymbol{v}_{1 \perp \text {,ran }}$ a Maxwellian distribution with 2-dimensional velocity dispersion $\sigma_{l}$, we obtain using also the definition for $g\left(v_{\mathrm{s} \perp}\right)$ given in Eq. (3)

$$
\begin{aligned}
& f\left(\boldsymbol{v}_{1 \perp}\right) v_{1 \perp} \mathrm{d} v_{1 \perp} \mathrm{d} \beta=\frac{1}{\left(\pi \sigma_{\mathrm{s}} \sigma_{1}\right)^{2}} \int_{0}^{2 \pi} \mathrm{d} \varphi \\
& \times \int_{0}^{\infty} \mathrm{e}^{-\frac{\left(v_{\perp \perp}-v_{\mathrm{s} \perp, \mathrm{rot})^{2}}\right.}{\sigma_{\mathrm{s}}^{2}}} v_{\mathrm{S} \perp} \mathrm{d} v_{\mathrm{S} \perp} \mathrm{e}^{-\frac{\left(v_{1 \perp}-w_{\perp}\right)^{2}}{\sigma_{1}^{2}}} v_{1 \perp} \mathrm{d} v_{1 \perp} \mathrm{d} \beta,
\end{aligned}
$$

where $\boldsymbol{w}_{\perp}=\boldsymbol{v}_{\perp \perp \text {,rot }}-\boldsymbol{v}_{\mathrm{t} \perp}$ and $\beta$ is the angle between $\boldsymbol{v}_{1 \perp}$ and $\boldsymbol{w}_{\perp}$.

We can then write the volume element, $\mathrm{d}^{3} x$, as

$\mathrm{d}^{3} x=\left(\boldsymbol{v}_{1 \perp} \cdot \hat{n}\right) \mathrm{d} t \mathrm{~d} S=v_{1 \perp} \cos \theta \mathrm{d} t \mathrm{~d} l \mathrm{~d} D_{\mathrm{ol}}$

$$
=v_{1 \perp} \cos \theta \mathrm{d} t R_{\mathrm{E}} \mathrm{d} u_{\mathrm{th}} \mathrm{d} \alpha \mathrm{d} D_{\mathrm{ol}},
$$

where $\theta$ is the angle between $\boldsymbol{v}_{1 \perp}$ and the normal, $\hat{n}$, to the lateral superficial element, $\mathrm{d} S=\mathrm{d} l \mathrm{~d} D_{\mathrm{ol}}$, of the microlensing tube, with $\mathrm{d} l=R_{\mathrm{E}} \mathrm{d} u_{\mathrm{th}} \mathrm{d} \alpha$ being the cylindrical segment of the tube ( $u_{\text {th }}$ is the threshold value for the impact parameter). Note that, if $\alpha$ is taken to be the angle between $\hat{n}$ and $\boldsymbol{w}_{\perp}$, it follows that $\theta=\alpha+\beta$, so that for a constant value of $\beta, \mathrm{d} \alpha=\mathrm{d} \theta$. Therefore, the microlensing differential event rate becomes

$\mathrm{d} \Gamma=n_{1}(\boldsymbol{x}, \mu) \mathrm{d} \mu f\left(\boldsymbol{v}_{1 \perp}\right) v_{1 \perp}^{2} \mathrm{~d} v_{1 \perp} \mathrm{d} \beta \cos \theta R_{\mathrm{E}} \mathrm{d} u_{\mathrm{th}} \mathrm{d} \alpha \mathrm{d} D_{\mathrm{ol}}$.

We assume, as usual, that the mass distribution of the lenses is independent of their position in M 31 or in the Galaxy (factorization hypothesis). So, the lens number density $n_{1}(\boldsymbol{x}, \mu)$ can be written as (Jetzer et al. 2002)

$n_{1}(\boldsymbol{x}, \mu)=\frac{\rho_{1}(\boldsymbol{x})}{\rho_{0}} \psi_{0}(\mu)$,

where $\rho_{0}$ is the local mass density in the Galaxy or the central density in $\mathrm{M} 31, \psi_{0}(\mu)$ the corresponding lens number density per unit of mass and the normalization is

$\int_{\mu_{\min }}^{\mu_{\mathrm{up}}} \psi_{0}(\mu) \mu \mathrm{d} \mu=\frac{\rho_{0}}{M_{\odot}}$.

Here $\mu_{\min }$ and $\mu_{\mathrm{up}}$ are the lower and the upper limits for the lens masses (see Sect. 3.3). Accordingly, the microlensing event rate is given by:

$$
\begin{aligned}
\Gamma\left(D_{\mathrm{os}}\right)= & \sqrt{\frac{4 G M_{\odot}}{c^{2}}} \int_{\mu_{\min }}^{\mu_{\mathrm{up}}} \mathrm{d} \mu \mu^{1 / 2} \psi_{0}(\mu) \int_{0}^{u_{\mathrm{T}}} \mathrm{d} u_{\mathrm{th}} \\
& \times \int_{0}^{D_{\mathrm{os}}} \mathrm{d} D_{\mathrm{ol}} \sqrt{\frac{D_{\mathrm{ol}}\left(D_{\mathrm{os}}-D_{\mathrm{ol}}\right)}{D_{\mathrm{os}}}} \frac{\rho_{\mathrm{l}}\left(D_{\mathrm{ol}}\right)}{\rho_{0}} \\
& \times \int_{0}^{2 \pi} \mathrm{d} \beta \int_{0}^{\infty} \mathrm{d} v_{1 \perp} f\left(\boldsymbol{v}_{1 \perp}\right) v_{1 \perp}^{2} \int_{-\pi / 2}^{+\pi / 2} \cos \theta \mathrm{d} \theta,
\end{aligned}
$$

where the integration on $\theta$ is performed between $-\pi / 2$ and $+\pi / 2$, since only lenses entering the microlensing tube are considered. After integrations on $\theta$ and $\beta$ we finally get

$$
\begin{aligned}
& \Gamma\left(D_{\mathrm{os}}\right)=2 \sigma_{\mathrm{l}} u_{\mathrm{T}} \sqrt{\frac{4 G M_{\odot}}{c^{2}}} \int_{\mu_{\mathrm{min}}}^{\mu_{\mathrm{up}}} \mathrm{d} \mu \mu^{1 / 2} \psi_{0}(\mu) \\
& \times \int_{0}^{D_{\mathrm{os}}} \mathrm{d} D_{\mathrm{ol}} \sqrt{\frac{D_{\mathrm{ol}}\left(D_{\mathrm{os}}-D_{\mathrm{ol}}\right)}{D_{\mathrm{os}}}} \frac{\rho_{\mathrm{l}}\left(D_{\mathrm{ol}}\right)}{\rho_{0}} \int_{0}^{\infty} P(z) \mathrm{d} z,
\end{aligned}
$$


where $z=v_{1 \perp} / \sigma_{1}$ and the function $P(z)$ is given by

$$
\begin{aligned}
P(z)= & \frac{2 \mathrm{e}^{-a^{2}}}{\pi} \int_{0}^{2 \pi} \mathrm{d} \varphi \int_{0}^{\infty} y \mathrm{e}^{-\left(y^{2}-2 a y \cos \varphi+\eta^{2}\right)} \mathrm{d} y \\
& \times z^{2} \mathrm{e}^{-z^{2}} I_{0}(2 \eta z) .
\end{aligned}
$$

Here $a\left(D_{\mathrm{os}}\right)=v_{\mathrm{s} \perp \text {,rot }} / \sigma_{\mathrm{s}}, y=v_{\mathrm{s} \perp} / \sigma_{\mathrm{s}}, \eta\left(D_{\mathrm{os}}, y, \varphi\right)=w_{\perp} / \sigma_{1}$ and $I_{0}(2 \eta z)$ is the zero-order modified Bessel function of the argument $2 \eta z$.

Note that $\chi(z)=z^{-1} P(z)$ is the dimensionless form of Eq. (5) and that it is properly normalized as it can be easily verified by using twice the relation $\int_{0}^{\infty} \mathrm{d} t \exp \left(-t^{2}\right) t I_{0}(2 q t)=$ $\exp \left(q^{2}\right) / 2$.

To take into account the source distribution in the M 31 bulge and disk, Eq. (11) has to be integrated not only over the distance of the lenses but also over the distance of the sources. Accordingly, the microlensing rate becomes

$\Gamma(x, y)=\frac{\int_{0}^{\infty} \rho_{\mathrm{s}}\left(D_{\mathrm{os}}\right) \Gamma\left(D_{\mathrm{os}}\right) \mathrm{d} D_{\mathrm{os}}}{\int_{0}^{\infty} \rho_{\mathrm{s}}\left(D_{\mathrm{os}}\right) \mathrm{d} D_{\mathrm{os}}}$,

where $x$ and $y$ are coordinates in the plane orthogonal to line of sight, $\rho_{\mathrm{s}}$ is the source mass density (which is the sum of the sources in the M31 bulge and disk).

Moreover, we compute the average Einstein time (which depends on the line of sight position given by the coordinates $x$ and $y$ ) as

$\left\langle t_{E}\right\rangle=\frac{\int_{0}^{\Gamma} t_{E} \mathrm{~d} \Gamma}{\Gamma}$.

\subsection{Pixel lensing basics}

Pixel lensing technique is based on the observation of the flux variations of every element (pixel) of an image (Ansari et al. 1997). Looking towards M31 a large number of stars contribute at the same time to the flux received by each pixel so that only highly magnified events can be detected.

To be detectable a microlensing event must give rise to a substantial flux variation with respect to the background $N_{\mathrm{bl}}=$ $N_{\text {gal }}+N_{\text {sky }}$, which is the sum of the M 31 surface brightness and the sky contribution. The excess photon count per pixel due to an ongoing microlensing event is

$\Delta N_{\text {pix }}=N_{\text {bl }}\left[A_{\text {pix }}-1\right]=f_{\text {see }} N_{\mathrm{s}}[A(t)-1]$

where $N_{\mathrm{s}}$ is the source photon count in the absence of lensing, $A(t)$ is the source magnification factor due to lensing (see e.g. Griest 1991) and $f_{\text {see }}$ the fraction of the seeing disk contained in a pixel. Therefore, the expected number of photons in a pixel will be $N_{\text {pix }}=N_{\text {bl }}+\Delta N_{\text {pix }}$.

Of course, a pixel lensing event is detectable if the excess pixel photon count is greater than the threshold pixel noise $\sigma_{\mathrm{T}}$. Accordingly, by requiring the signal to be at least $3 \sigma_{\mathrm{T}}$ level above the baseline count, one obtains a threshold value for the amplification (Kerins et al. 2001)

$A_{\mathrm{T}}=1+\frac{3 \sigma_{\mathrm{T}}}{f_{\mathrm{see}} N_{\mathrm{s}}}$ which corresponds to a threshold value $u_{\mathrm{T}}$ for the impact parameter, via the well known relation between the lens impact parameter and the amplification factor.

One can estimate $\sigma_{\mathrm{T}}$ as the maximum between the statistical error $\propto \sqrt{N_{\mathrm{bl}}}$ and $\simeq 3 \times 10^{-3} N_{\mathrm{bl}}$ that is determined by the pixel flux stability. Accordingly, $u_{\mathrm{T}}$ depends on both the line of sight to M 31 and the source magnitude $M$.

Hence, by averaging on the source luminosity function $\phi(M)$, we can evaluate the average threshold impact parameter for any direction towards the M31 galaxy, so that we get

$\left\langle u_{\mathrm{T}}(x, y)\right\rangle_{\phi}=\frac{\int u_{\mathrm{T}}(x, y ; M) \phi(M) \mathrm{d} M}{\int \phi(M) \mathrm{d} M}$,

where the coordinates $x$ and $y$ span the sky plane towards M 31 .

By using the threshold impact parameter defined in Eq. (17), one obtains the pixel lensing rate as follows (Kerins et al. 2001, 2003; De Paolis et al. 2005)

$\Gamma_{p}(x, y)=\left\langle u_{T}(x, y)\right\rangle_{\phi} \Gamma(x, y)$.

\section{Modeling}

\subsection{M31 and Galaxy mass distribution models}

The M 31 disk, bulge and halo mass distributions are described adopting the reference model discussed in Kerins (2004). This model, providing remarkable good fits to the M31 surface brightness and rotation curve profiles, can be considered as an acceptable model for the mass distribution in the M 31 galaxy. Accordingly, the mass density of the M31 disk stars is described by a sech-squared profile

$\rho_{\mathrm{D}}(R, z)=\rho_{\mathrm{D}}(0) \exp (-R / h) \operatorname{sech}^{2}(z / H)$,

where $H=0.3 \mathrm{kpc}, h=6.4 \mathrm{kpc}$ and $\rho_{\mathrm{D}}(0)=0.35 \times$ $10^{9} M_{\odot} \mathrm{pc}^{-3}$ are, respectively, the scale height and scale lengths of the disk and the disk central mass density. $R$ is the distance on the M 31 disk plane (described by the coordinates $x$ and $y$ ) and $z$ is the distance from it. The M 31 disk is assumed to be inclined by an angle $i=77^{\circ}$ and the azimuthal angle relative to the near minor axis is $\phi=-38.6^{\circ}$.

The M 31 bulge is parameterized by a flattened power law of the form

$\rho_{\mathrm{B}}(R, z)=\rho_{\mathrm{B}}(0)\left[1+\left(\frac{R}{\tilde{a}}\right)^{2}+q^{-2}\left(\frac{z}{\tilde{a}}\right)^{2}\right]^{-s / 2}$,

where $\rho_{\mathrm{B}}(0) \simeq 4.5 \times 10^{9} M_{\odot} \mathrm{kpc}^{-3}, q \simeq 0.6$ is the ratio of the minor to major axis, $\tilde{a} \simeq 1 \mathrm{kpc}$ and $s \simeq 3.8$. Both the M 31 disk and bulge are truncated at a distance $R=40 \mathrm{kpc}$.

We remark that the twisting of the optical isophotes in the inner M31 regions indicates that the bulge major axis is offset by $\simeq 15^{\circ}$ from the disk major axis (Stark \& Binney 1994). The consideration of this effect by Kerins et al. (2005) leads to the evaluation of pixel lensing rates that show spatial distributions tilted of the same amount inside 5 arcmin from the M 31 center. The twisting effect vanishes at larger distances due to the increasing contribution to microlensing by M31 disk and 


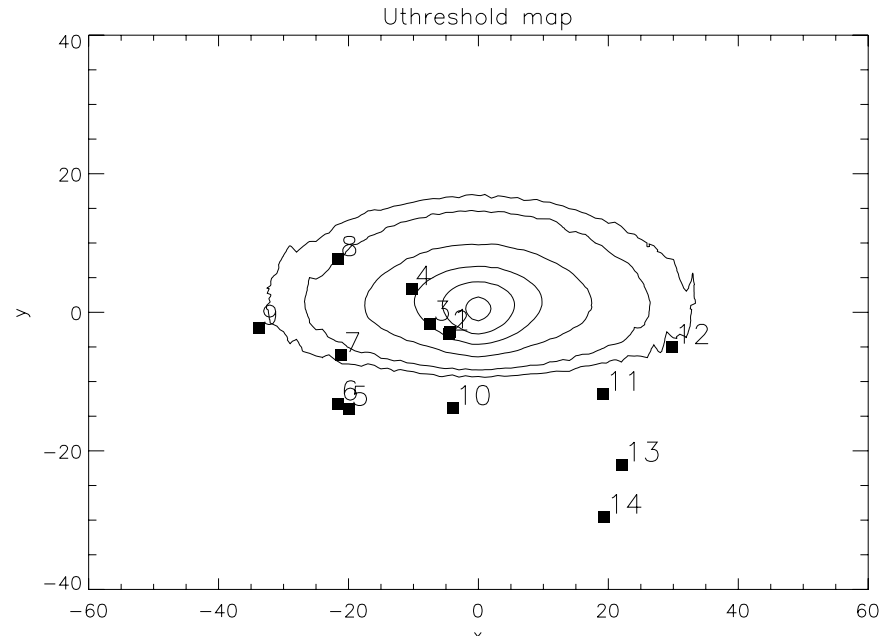

Fig. 1. The $\left\langle u_{\mathrm{T}}(x, y)\right\rangle_{\phi_{\mathrm{RG}}}$ contour plot towards the M31 galaxy is shown with the position in the sky plane of the 14 MEGA candidate microlensing events. From the inner to the outer part of the figure $\left\langle u_{\mathrm{T}}(x, y)\right\rangle_{\phi_{\mathrm{RG}}}$ increases with lines referring to the values 0.02 , $0.04,0.06,0.08$ and 0.10 , respectively. Outside the 0.10 contour line $\left\langle u_{\mathrm{T}}(x, y)\right\rangle_{\phi_{\mathrm{RG}}}$ is almost constant. Note that the positions of the events 1 and 2 are almost on top of each other. The same is true as well for all other figures.

halo. Clearly, our results in Figs. 1-3 do not show the above mentioned effect since here we are considering only a flattened bulge without twist. However, we expect that the consideration of the isophote twisting does not substantially modify our results about both MACHO-to-self lensing probability and event time scale ratios (given in Tables 2-5), particularly for events at large distance from the M 31 center.

The dark matter in the M31 halo is assumed to follow an isothermal profile

$\rho_{\mathrm{H}}(r)=\rho_{\mathrm{H}}(0) \frac{a^{2}}{a^{2}+r^{2}}$,

with core radius $a=4 \mathrm{kpc}$ and central dark matter density $\rho_{\mathrm{H}}(0)=6.5 \times 10^{7} M_{\odot} \mathrm{kpc}^{-3}$. The M31 halo is truncated at $100 \mathrm{kpc}$ with asymptotic rotational velocity $v_{\text {rot }} \simeq$ $235 \mathrm{~km} \mathrm{~s}^{-1}$.

We do not consider other dark matter distribution models, as King-Michie (Binney \& Tremaine 1987) or NFW (Navarro et al. 1997) suggested by $N$ body simulations. The effect of using such models, which have a more concentred dark mass distribution, is both to decrease the spatial distribution of MACHOs at large distance from the M 31 center, where the rotation curve is poorly determined, and to increase it in the innermost region, where the MACHO contribution is relatively unimportant with respect to that of bulge and disk. Regarding the former aspect, we also note that in the MEGA experiment the typical MACHO lens distance (about $20 \mathrm{kpc}$ ) is too small to appreciate the effect of this choice. Overall, the current data do not allow one to perform a meaningfully fine tuning of the dark matter parameters.

As usual, the mass density profile for the MW disk is described with a double exponential profile

$\rho_{\mathrm{D}}(R, z)=\rho_{\mathrm{D}}\left(R_{0}\right) \exp \left(-\left(R-R_{0}\right) / h\right) \exp (-|z| / H)$,

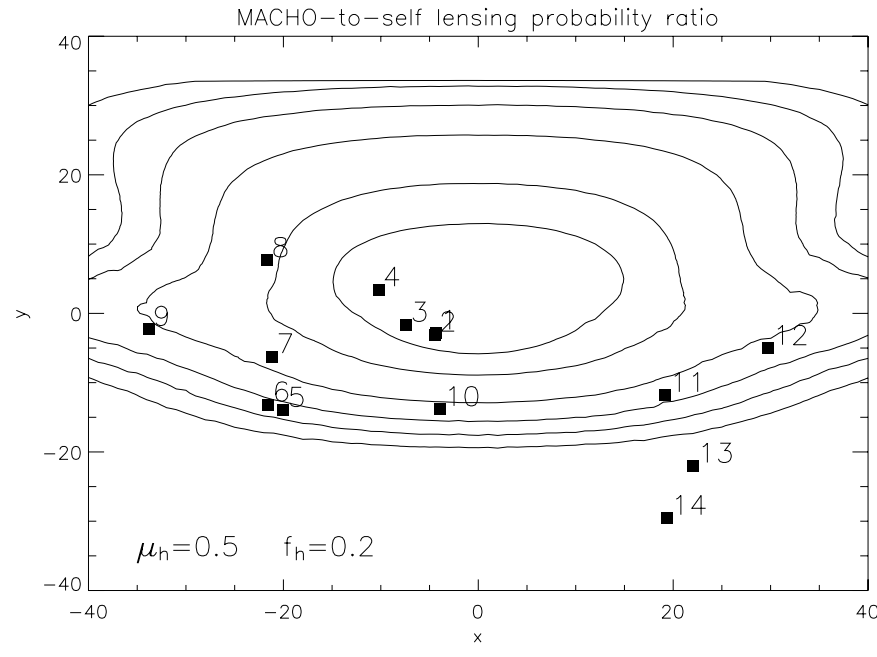

Fig. 2. MACHO-to-self lensing probability ratio $\left(P_{\mathrm{h}} / P_{\mathrm{s}}\right)_{\mathrm{An}}$ map projected onto the sky plane. Here and in the following figures we assume a fraction $f_{\mathrm{h}}=20 \%$ of dark matter and a MACHO mass $\mu_{\mathrm{h}}=0.5$, both in M 31 and MW halos. From the inner to the outer part $\left(P_{\mathrm{h}} / P_{\mathrm{s}}\right)_{\mathrm{An}}$ increases with lines referring to the values $0.5,1,2,3,4$ and 5 , respectively.

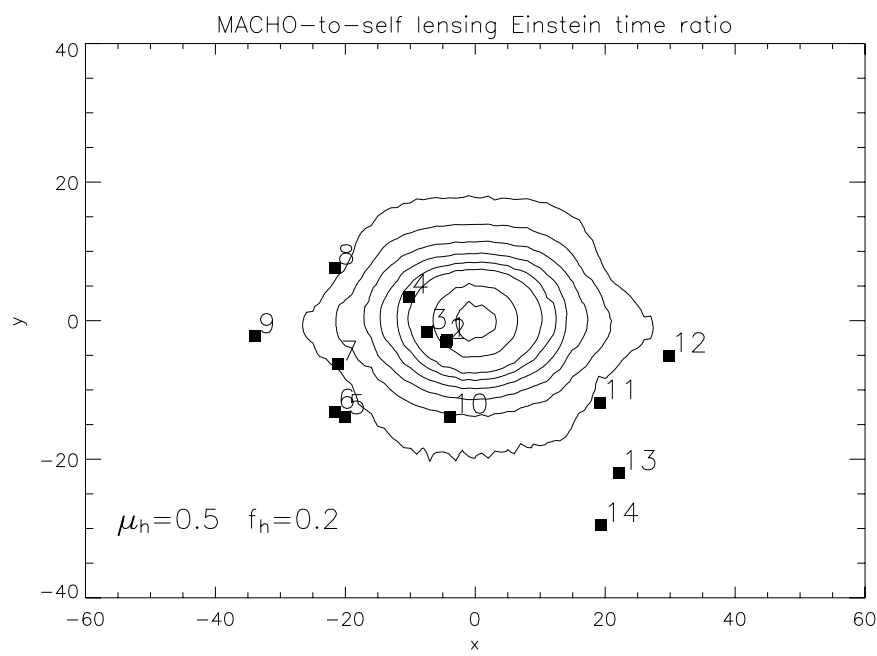

Fig. 3. The MACHO-to-self lensing Einstein time ratio $\left\langle t_{\mathrm{E}} \mathrm{h}\right\rangle /\left\langle t_{\mathrm{E}}\right\rangle$ map is given for $\mu_{\mathrm{h}}=0.5$. Going from the inner to the outer part the ratio decreases with lines corresponding to values in between 2 to 1.25 with a step of 0.25 .

with Earth position from the Galactic center at $R_{0} \simeq 8.5 \mathrm{kpc}$, scale height $H \simeq 0.3 \mathrm{kpc}$, scale length $h \simeq 3.5 \mathrm{kpc}$ and local mass density $\rho_{\mathrm{D}}\left(R_{0}\right) \simeq 1.67 \times 10^{8} M_{\odot} \mathrm{kpc}^{-3}$.

The dark halo in our Galaxy is also assumed to follow an isothermal profile with core radius $a \simeq 5.6 \mathrm{kpc}$ and local dark matter density $\rho_{\mathrm{H}}\left(R_{0}\right) \simeq 1.09 \times 10^{7} M_{\odot} \mathrm{kpc}^{-3}$. The corresponding asymptotic rotational velocity is $v_{\text {rot }} \simeq 220 \mathrm{~km} \mathrm{~s}^{-1}$. The MW halo is truncated at $R \simeq 100 \mathrm{kpc}$.

For both M 31 and MW halos, the fraction of dark matter in form of MACHOs is assumed to be $f_{\mathrm{h}} \simeq 0.2$ (Alcock et al. 2000). However, most of our results can easily be rescaled to get the corresponding figures for other values of $f_{\mathrm{MACHO}}$. 
Moreover, we assume that the random velocities of stars and MACHOs follow Maxwellian distributions with onedimensional velocity dispersion $\sigma=30,100,166 \mathrm{~km} \mathrm{~s}^{-1}$ and $30,156 \mathrm{~km} \mathrm{~s}^{-1}$ for the M 31 disk, bulge, halo and MW disk and halo, respectively. In addition, a M 31 bulge rotational velocity of $30 \mathrm{~km} \mathrm{~s}^{-1}$ has been taken into account (Kerins et al. 2001; An et al. 2004).

\subsection{Stellar luminosity function}

Pixel lensing event detection by the MEGA collaboration is performed in the red band ${ }^{1}$ and, thus, red giants are the most luminous stars in this band. Therefore, we may safely assume that the overwhelming majority of the pixel lensing event sources are red giants.

Moreover, in the lack of precise information about the stellar luminosity function in M31, we adopt the luminosity function derived from the stars in the Galaxy and assume that it also holds for M 31 .

Accordingly, following (Mamon \& Soneira 1982) we assume that the stellar luminosity function does not depend on the position and in the magnitude range $-6 \leq M \leq 16$ it is proportional to the expression

$\phi_{*}(M) \propto \frac{10^{\beta\left(M-M^{*}\right)}}{\left[1+10^{-(\alpha-\beta) \delta\left(M-M^{*}\right)}\right]^{1 / \delta}}$,

where, in the red band, $M^{*}=1.4, \alpha \simeq 0.74, \beta=0.045$ and $\delta=1 / 3$. Moreover, the fraction of red giants (over the total star number) as a function of $M$ is approximated as (Mamon \& Soneira 1982)

$$
\begin{aligned}
f_{\mathrm{RG}}(M) & =1-C \exp \left[\alpha(M+\beta)^{\gamma}\right] & & \text { for }-6 \leq M \leq 3 \\
& =0 & & \text { for } M \geq 3,
\end{aligned}
$$

where, in the red band, $C \simeq 0.31, \alpha \simeq 6.5 \times 10^{-4}, \beta=7.5$ and $\gamma \simeq 3.2$.

Therefore, the red giant luminosity function will be $\phi_{\mathrm{RG}}(M) \propto \phi_{*}(M) \times f_{\mathrm{RG}}(M)$ and the fraction of red giants averaged on the magnitude is given by

$\left\langle f_{\mathrm{RG}}\right\rangle=\frac{\int_{-6}^{3} \phi_{\mathrm{RG}}(M) \mathrm{d} M}{\int_{-6}^{16} \phi_{*}(M) \mathrm{d} M} \simeq 5.3 \times 10^{-3}$,

from which it follows that the local number density of red giants will be $n_{\mathrm{RG}} \simeq 5.3 \times 10^{-3} n_{*}$, where $n_{*}$ is the local stellar number density.

\subsection{Mass functions}

As concerns the lens mass function $\psi_{0}(\mu)$ in Eqs. (8)-(11), for lenses belonging to the bulge and disk star populations, the lens mass is assumed to follow a broken power law (Gould et al. 1997)

$$
\begin{aligned}
& \psi_{0}(\mu)=K \mu^{-0.56} \text { for } 0.1 \leq \mu \leq 0.59 \\
& =K \mu^{-2.20} \text { for } 0.59 \leq \mu \leq \mu_{\text {up }}
\end{aligned}
$$

${ }^{1}$ Indeed, observations in the red band, by minimizing light absorption in M31 and MW disks by the intervening dust, offer the best compromise between sampling and sky background. Observations in other bands $(B$ and $V$ ) are commonly used to test achromaticity of the candidate events.
Table 2. MACHO-to-self lensing probability ratio $\left(P_{\mathrm{h}} / P_{\mathrm{s}}\right)_{\mathrm{An}}$ towards the 14 MEGA events are given for different MACHO mass values.

\begin{tabular}{ccccc}
\hline \hline MEGA & $\mu_{\mathrm{h}}=0.01$ & 0.1 & 0.5 & 1 \\
\hline 1 & 1.65 & 0.52 & 0.23 & 0.16 \\
2 & 1.76 & 0.56 & 0.25 & 0.18 \\
3 & 2.22 & 0.71 & 0.31 & 0.22 \\
4 & 2.52 & 0.78 & 0.35 & 0.24 \\
5 & 24.58 & 7.72 & 3.46 & 2.40 \\
6 & 23.46 & 7.53 & 3.38 & 2.40 \\
7 & 12.93 & 3.83 & 1.80 & 1.26 \\
8 & 9.79 & 3.15 & 1.40 & 0.96 \\
9 & 19.49 & 6.15 & 2.79 & 1.99 \\
10 & 17.19 & 5.44 & 2.42 & 1.73 \\
11 & 19.22 & 6.04 & 2.74 & 1.88 \\
12 & 18.91 & 5.88 & 2.62 & 1.91 \\
13 & 54.13 & 17.60 & 7.87 & 5.67 \\
14 & 112.32 & 35.47 & 17.07 & 11.12 \\
\hline
\end{tabular}

Table 3. MACHO-to-self lensing Einstein time ratio $\left\langle t_{\mathrm{E}}\right\rangle /\left\langle t_{\mathrm{E}}\right\rangle$ towards the 14 MEGA events are given for different MACHO mass values.

\begin{tabular}{ccccc}
\hline \hline MEGA & $\mu_{\mathrm{h}}=0.01$ & 0.1 & 0.5 & 1 \\
\hline 1 & 0.30 & 0.96 & 2.15 & 3.02 \\
2 & 0.31 & 1.00 & 2.42 & 3.24 \\
3 & 0.29 & 0.94 & 2.02 & 2.91 \\
4 & 0.20 & 0.65 & 1.47 & 2.12 \\
5 & 0.16 & 0.51 & 1.10 & 1.59 \\
6 & 0.16 & 0.49 & 1.09 & 1.55 \\
7 & 0.15 & 0.52 & 1.10 & 1.58 \\
8 & 0.13 & 0.41 & 0.92 & 1.35 \\
9 & 0.13 & 0.42 & 0.97 & 1.34 \\
10 & 0.17 & 0.54 & 1.21 & 1.69 \\
11 & 0.16 & 0.50 & 1.11 & 1.61 \\
12 & 0.14 & 0.44 & 0.98 & 1.36 \\
13 & 0.16 & 0.49 & 1.08 & 1.51 \\
14 & 0.15 & 0.48 & 1.03 & 1.52 \\
\hline
\end{tabular}

where the lower limit $\mu_{\min }=0.1$ and the upper limit $\mu_{\text {up }}$ is 1 for M 31 bulge stars and 10 for M 31 and MW disk stars (see also Kerins et al. 2001). $K$ is fixed according to the normalization given by Eq. (9). The resulting mean mass for lenses in the bulges and disks are $\left\langle m_{\mathrm{b}}\right\rangle \sim 0.37 M_{\odot}$ and $\left\langle m_{\mathrm{d}}\right\rangle \sim 0.69 M_{\odot}$, respectively.

For the lens mass in the M31 and MW halos we assume the $\delta$-function approximation

$\psi_{0}(\mu)=\frac{\rho_{0}}{M_{\odot} \mu_{\mathrm{h}}} \delta\left(\mu-\mu_{\mathrm{h}}\right)$

and we take a MACHO mass, in solar units, $\mu_{\mathrm{h}}=$ $10^{-2}, 10^{-1}, 0.5,1$. 
Table 4. For the MC revealed events (which pass the MEGA selection criteria), the median values $\mu_{*}^{\text {median }}$ (Col. 2) of the lens star mass distribution for self-lensing events, $t_{1 / 2}^{\text {median }}\left(\right.$ Cols. 3-7) and $R_{\max }^{\text {median }}($ Cols. 8-12) for self-lensing and MACHO-lensing are given towards the 14 MEGA events.

\begin{tabular}{|c|c|c|c|c|c|c|c|c|c|c|c|}
\hline \multirow[b]{3}{*}{ MEGA } & \multirow{3}{*}{$\begin{array}{c}\mu_{*}^{\text {median }} \\
\text { self }\end{array}$} & \multicolumn{5}{|c|}{$t_{1 / 2}^{\text {median }}$} & \multicolumn{5}{|c|}{$R_{\max }^{\text {median }}$} \\
\hline & & \multirow[t]{2}{*}{ self } & \multicolumn{3}{|c|}{ MACHO } & \multirow[b]{2}{*}{1} & \multirow[t]{2}{*}{ self } & \multicolumn{4}{|c|}{ MACHO } \\
\hline & & & $\mu_{\mathrm{h}}=0.01$ & 0.1 & 0.5 & & & $\mu_{\mathrm{h}}=0.01$ & 0.1 & 0.5 & 1 \\
\hline 1 & 0.48 & 15.66 & 4.96 & 11.22 & 19.51 & 22.49 & 21.56 & 21.16 & 21.45 & 21.65 & 21.61 \\
\hline 2 & 0.48 & 15.33 & 5.06 & 11.22 & 18.15 & 22.04 & 21.62 & 21.31 & 21.52 & 21.64 & 21.70 \\
\hline 3 & 0.49 & 16.38 & 5.17 & 11.20 & 19.32 & 22.62 & 21.69 & 21.41 & 21.64 & 21.76 & 21.74 \\
\hline 4 & 0.62 & 18.16 & 5.04 & 11.43 & 18.81 & 23.04 & 21.87 & 21.57 & 21.85 & 21.93 & 21.94 \\
\hline 5 & 0.46 & 20.62 & 4.85 & 11.43 & 19.80 & 23.59 & 22.22 & 21.85 & 22.11 & 22.23 & 22.22 \\
\hline 6 & 0.46 & 21.43 & 4.77 & 11.57 & 19.88 & 24.27 & 22.17 & 21.85 & 22.11 & 22.23 & 22.23 \\
\hline 7 & 0.50 & 20.72 & 4.95 & 10.97 & 19.12 & 22.96 & 22.10 & 21.79 & 22.02 & 22.14 & 22.19 \\
\hline 8 & 0.65 & 21.47 & 5.15 & 11.60 & 19.90 & 23.65 & 22.13 & 21.81 & 22.05 & 22.18 & 22.21 \\
\hline 9 & 0.57 & 24.04 & 5.20 & 12.21 & 19.94 & 24.17 & 22.19 & 21.85 & 22.08 & 22.18 & 22.20 \\
\hline 10 & 0.45 & 20.07 & 4.73 & 10.79 & 18.98 & 23.09 & 22.19 & 21.83 & 22.06 & 22.20 & 22.24 \\
\hline 11 & 0.46 & 20.37 & 4.88 & 11.02 & 19.79 & 23.66 & 22.14 & 21.86 & 22.08 & 22.21 & 22.22 \\
\hline 12 & 0.52 & 22.30 & 5.00 & 11.77 & 20.24 & 23.94 & 22.17 & 21.84 & 22.05 & 22.20 & 22.21 \\
\hline 13 & 0.44 & 22.58 & 4.98 & 12.24 & 21.14 & 25.28 & 22.32 & 21.99 & 22.20 & 22.34 & 22.35 \\
\hline 14 & 0.43 & 24.15 & 5.34 & 12.58 & 21.90 & 26.53 & 22.34 & 22.03 & 22.25 & 22.35 & 22.38 \\
\hline
\end{tabular}

Table 5. MACHO-to-self lensing probability ratios $\left(P_{\mathrm{h}} / P_{\mathrm{s}}\right)_{\mathrm{MC}}$ towards the 14 observed MEGA events are given for different MACHO mass values. Probabilities are now calculated from Eq. (35) by considering microlensing rates, $t_{1 / 2}$ and $R_{\max }$ distributions for the MC revealed events. The results in the table scale with the MACHO fraction value as $f_{\mathrm{h}} / 0.2$.

\begin{tabular}{ccccc}
\hline \hline MEGA & $\mu_{\mathrm{h}}=0.01$ & 0.1 & 0.5 & 1 \\
\hline 1 & 3.65 & 0.70 & 0.18 & 0.10 \\
2 & 5.71 & 0.79 & 0.16 & 0.08 \\
3 & 6.70 & 1.06 & 0.23 & 0.14 \\
4 & 0.36 & 0.56 & 0.33 & 0.24 \\
5 & 63.51 & 13.41 & 3.64 & 1.98 \\
6 & 7.56 & 6.56 & 3.48 & 2.35 \\
7 & 0.45 & 1.83 & 1.46 & 3.04 \\
8 & 2.67 & 2.88 & 1.46 & 0.97 \\
9 & 109.47 & 14.32 & 3.58 & 1.74 \\
10 & 0.98 & 1.95 & 1.98 & 1.79 \\
11 & 62.95 & 8.29 & 2.56 & 1.28 \\
12 & 0.04 & 0.55 & 1.36 & 1.79 \\
13 & 20.81 & 23.20 & 9.44 & 4.98 \\
14 & 26.99 & 29.88 & 18.50 & 12.24 \\
\hline
\end{tabular}

\section{Analytical results}

In the present analysis we adopt the parameters for the INT and the Sloan-r filter on the WFC (Wide-Field Camera) used by the MEGA collaboration (de Jong et al. 2004). The Telescope diameter, the pixel field of view and the image exposition time are $2.5 \mathrm{~m}, 0.33$ arcsec and $t_{\exp }=760 \mathrm{~s}$, respectively. We also use a gain or conversion factor of $2.8 \mathrm{e}^{-} / \mathrm{ADU}$, and a loss factor $\simeq 3$, for both atmospheric and instrumental. The zero-point with the Sloan-r WFC turns out to be $\sim 24.3 \mathrm{mag} \operatorname{arcsec}^{-2}$ (Belokurov et al. 2005). Moreover, we adopt a value $\simeq 1.5$ arcsec for the average seeing conditions, a sky background $m_{\text {sky }} \simeq 20.9$ mag $\operatorname{arcsec}^{-2}$ (corresponding to a Moon eclipse) and a minimum noise level of $\sim 2.5 \times 10^{-3} N_{\mathrm{bl}} . N_{\mathrm{bl}}$ is the baseline photon count which is the sum of the M31 surface brightness given by Kent (1989) and the sky contribution.

Maps of optical depth, expected event number and event timescale in pixel lensing experiments have been presented in a previous paper (De Paolis et al. 2005) together with the study of the dependence on microlensing quantities with the assumed M 31 mass distribution model (see also Kerins 2004). Our results are also in good agreement with previous analytical estimates for the rate, the timescale distribution and the optical depth (Baltz et al. 2003; Gyuk \& Crotts 2000).

In Fig. 1 the map of $\left\langle u_{\mathrm{T}}(x, y)\right\rangle_{\phi_{\mathrm{RG}}}$ shows that in the central M31 regions the lens impact parameter on average is $\leq 0.04 \mathrm{im}$ plying that only high magnified events with $A_{\mathrm{T}} \geq 25$ are in principle detectable. The asymmetrical shape is due to the internal extinction of the M31 disk for which we use the value 0.74 mag given by Kent (1989). Indeed, due to the inclination of the M 31 disk, along a line of sight towards the southern region there exists a larger number of source stars (with respect to the corresponding northern field) which are not absorbed by the M 31 disk dust (and therefore appear with a smaller magnitude), lying at larger averaged values for $u_{\mathrm{T}}$.

In Fig. 2, assuming a MACHO mass $\mu_{\mathrm{h}}=0.5$ and a halo MACHO fraction $f_{\mathrm{h}}=0.2$, we show the map of the MACHO-to-self lensing probability ratio $\left(P_{\mathrm{h}} / P_{\mathrm{s}}\right)_{\mathrm{An}}$. We find that in the M31 central regions microlensing is dominated by self-lensing contributions while MACHO-lensing becomes important at distances $>10$ arcmin from the center. In the figure 
it is also evident the well known near-far disk asymmetry due to the inclination of the M 31 disk (Crotts 1992; Baillon et al. 1993; Jetzer 1994).

In Table 2, we show how the MACHO-to-self lensing probability ratio $\left(P_{\mathrm{h}} / P_{\mathrm{s}}\right)_{\mathrm{An}}$ depends on the MACHO mass $\mu_{\mathrm{h}}$ towards the 14 MEGA events. The general trend is that $\left(P_{\mathrm{h}} / P_{\mathrm{s}}\right)_{\mathrm{An}}$ increases as $\mu_{\mathrm{h}}$ decreases as a consequence of the increase of the MACHO number density. Moreover, for a given value of $\mu_{\mathrm{h}}$, $\left(P_{\mathrm{h}} / P_{\mathrm{s}}\right)_{\mathrm{An}}$ increases with the distance from the M 31 center.

In Fig. 3 the map of the MACHO-to-self lensing Einstein time ratio $\left(t_{\mathrm{E} \mathrm{h}} / t_{\mathrm{E} \mathrm{s}}\right)_{\mathrm{An}}$ is given for a MACHO mass of $0.5 M_{\odot}$. In the region inside $\simeq 5$ arcmin from the $\mathrm{M} 31$ center microlensing events due to MACHOs have twice as long a duration as compared to self-lensing events, while in the regions far away from the M 31 center all events have roughly the same duration. Obviously, the MACHO-to-self lensing Einstein time ratio depends on the assumed MACHO mass. This fact is clearly seen in Table 3, where the ratio $\left(\left\langle t_{\mathrm{E}}\right\rangle /\left\langle t_{\mathrm{E}}\right\rangle\right)_{\mathrm{An}}$ towards the 14 MEGA events is given for different MACHO mass values. $\mu_{\mathrm{h}}=0.5$ corresponds to the MACHO mass value for which the 10 outer MEGA events (5-14) are characterized by having a MACHO-to-self lensing Einstein time ratio equal to unity, i.e., events due to either MACHO-lensing or self-lensing have roughly the same duration. Thus, these different types of events are indistinguishable on the basis of their timescale alone. The situation is instead much more favorable for MACHO masses smaller or larger than $0.5 M_{\odot}$.

We would like to emphasize that in pixel lensing experiments $t_{\mathrm{E}}$ is not a directly observable quantity, since the relevant time scale is the full-width half-maximum event duration $t_{1 / 2}$, which depends on $t_{E}$ and the impact parameter $u_{0}$ (Gondolo 1999). However, since the probability for a given $u_{0}$ value is practically the same for self-lensing and MACHO-lensing events (as we have verified by using the MC code) the ratio $\left(\left\langle t_{E \mathrm{~h}}\right\rangle /\left\langle t_{E \mathrm{~s}}\right\rangle\right)_{\mathrm{An}}$ is equivalent to the ratio $\left(\left\langle t_{1 / 2 \mathrm{~h}}\right\rangle /\left\langle t_{1 / 2} \mathrm{~s}\right\rangle\right)_{\mathrm{An}}$, at least for the MC generated events. Of course, as it will be more clear in the following sections (in particular from Table 4 and Fig. 10), it does not mean that the same conclusion holds for the MC revealed events, since the (normalized) event number which pass the MEGA selection criteria turns out to depend on $t_{1 / 2}$ and $R_{\max }$ (see Fig. 6).

\section{Monte Carlo simulation}

Once the event location (one of the 14 MEGA events towards M 31) has been selected, for any lens and source population present along the line of sight we have to make as next the choice over the following five parameters: source distance $D_{\mathrm{os}}$, lens distance $D_{\mathrm{ol}}$, lense effective transverse velocity $v_{1 \perp}$, lens mass $\mu$ and source magnitude $M$. We shall denote these parameters by $x_{i}$, with $i=1, \ldots, 5$ in the order just listed (e.g. $x_{1}=D_{\text {os }}$, etc.). The probability with which we select the events according to one of the parameters is then given by

$P\left(x_{i}\right) \mathrm{d} x_{i}=\frac{1}{N_{\mathrm{ev}}} \frac{\partial N_{\mathrm{ev}}}{\partial x_{i}} \mathrm{~d} x_{i}$

where $\frac{\partial N_{\text {ev }}}{\partial x_{i}}$ is defined as being the integrand of the event number $N_{\mathrm{ev}}$ integrated over all variables $x_{j}$ without, however, the considered variable $x_{i}$. Clearly when integrating $\frac{\partial N_{\mathrm{ev}}}{\partial x_{i}}$ over $\mathrm{d} x_{i}$ we obtain again $N_{\mathrm{ev}}$.

This way, for instance, for self-lensing events the probability of extracting a lens with mass $\mu$ turns out to be

$P\left(x_{4}=\mu\right) \mathrm{d} \mu \propto \mu^{1 / 2} \psi_{0}(\mu) \mathrm{d} \mu$,

where $\psi_{0}(\mu)$ is the lens number density distribution defined in Eq. (26).

The probability of a source ${ }^{2}$ at distance $D_{\text {os }}$ and a lens at distance $D_{\mathrm{ol}}$ from the observer, respectively, is given by

$P\left(D_{\mathrm{os}}\right) \mathrm{d} D_{\mathrm{os}} \propto \rho_{\mathrm{s}}\left(D_{\mathrm{os}}\right) D_{\mathrm{os}}^{3 / 2}\left(\int_{0}^{D_{\mathrm{os}}} P\left(D_{\mathrm{ol}}\right) \mathrm{d} D_{\mathrm{ol}}\right) \mathrm{d} D_{\mathrm{os}}$

and

$P\left(D_{\mathrm{ol}}\right) \mathrm{d} D_{\mathrm{ol}} \propto \rho_{\mathrm{l}}\left(D_{\mathrm{ol}}\right)\left[D_{\mathrm{ol}}\left(D_{\mathrm{os}}-D_{\mathrm{ol}}\right)\right]^{1 / 2} \mathrm{~d} D_{\mathrm{ol}}$,

where $\rho_{\mathrm{l}}\left(D_{\mathrm{ol}}\right)$ and $\rho_{\mathrm{s}}\left(D_{\mathrm{os}}\right)$ are, respectively, the lens and the source mass densities.

The probability of extracting a source with magnitude $M$ is weighted by the function

$P(M) \mathrm{d} M \propto u_{\mathrm{T}}(M) \phi_{\mathrm{RG}}(M) \mathrm{d} M$,

where $\phi_{\mathrm{RG}}(M)$ is the source magnitude distribution for red giants defined by means of Eqs. (23)-(24) and, through $u_{\mathrm{T}}(M)$, we take into account the surface brightness variation along the field of view. Indeed, we find that the median value of the source magnitude distribution decreases from $M^{\text {median }}=-2.18$ for the innermost MEGA directions to $M^{\text {median }}=-1.18$ for the outermost ones. In Fig. 4 we show the distribution of the source absolute magnitude for all MC generated events and for the revealed (see after) events towards the direction of the MEGA 7 event. For a given source and lens position the probability of extracting a lens with transverse velocity $v_{\perp \perp}$ is given by Eq. (12), where $z=v_{1 \perp} / \sigma_{1}$. In Fig. 5, for both disk-disk and bulge-disk events, we show the lens transverse velocity distributions for MC generated events towards the MEGA 1 direction. The shorter $\left\langle v_{\perp \perp}\right\rangle$ value in the first case will lead to an increase in the event timescale.

The probability of extracting an event with impact parameter $u_{0}$ is constant for values of $u_{0}$ in the interval in between 0 and $u_{\mathrm{T}}$, as defined in Eq. $(17)^{3}$. Thus it is assumed that

$P\left(u_{0}\right) \propto$ const.

Once all the parameters have been fixed, for each event we build the corresponding lightcurve using the same time sampling of the MEGA campaign and the same observing and instrumental conditions of the considered experiment. We add a Gaussian noise modulated by the Moon phase and use a Paczyński fit (Paczyński 1986) to evaluate the microlensing parameters.

The selection of the MC generated events is based on the same criteria adopted by the MEGA collaboration. By using

\footnotetext{
${ }^{2}$ Here we take into account that the source number inside the pixel solid angle increases with the distance as $D_{\mathrm{os}}^{2}$.

3 In order to avoid a sharp cutoff in several obtained plots (see, e.g., Fig. 7) we allow for events with $u_{0} \gtrsim u_{\mathrm{T}}$ to be detected.
} 


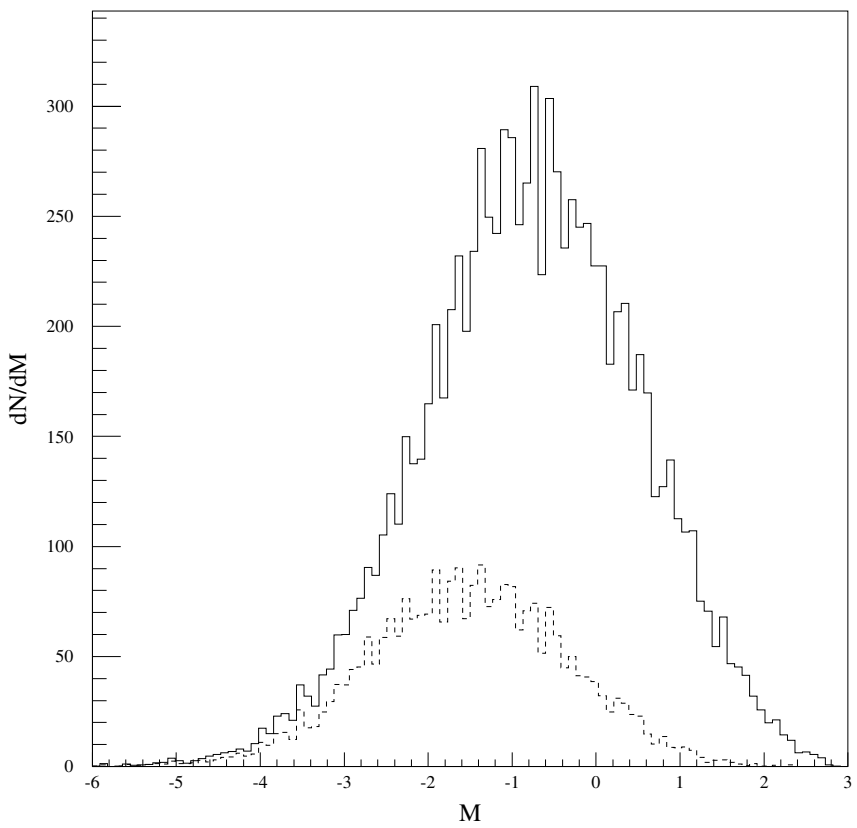

Fig. 4. Plot of the source absolute magnitude distribution for the generated events (solid line) and for the revealed events (dashed line) towards the MEGA 7 direction.



Fig. 5. The lens transverse velocity $v_{1 \perp}$ distribution for the MC generated events towards the MEGA 1 direction is shown in the case of disk-disk (solid line) and bulge-disk events (dashed line).

the results of the Paczyński fit we filter the lightcurves with the following selection criteria: peak sampling, peak significance, peak width, baseline flatness and goodness of fit (for more details see Appendix A.1 in de Jong et al. 2004). The criteria that more severely cut the MC generated events are the peak sampling and the peak significance, which depends through the evaluation of the statistical errors on the the Moon phase. The former criterium leads to select events with well sampled lightcurves (within the MEGA campaign), the latter to events

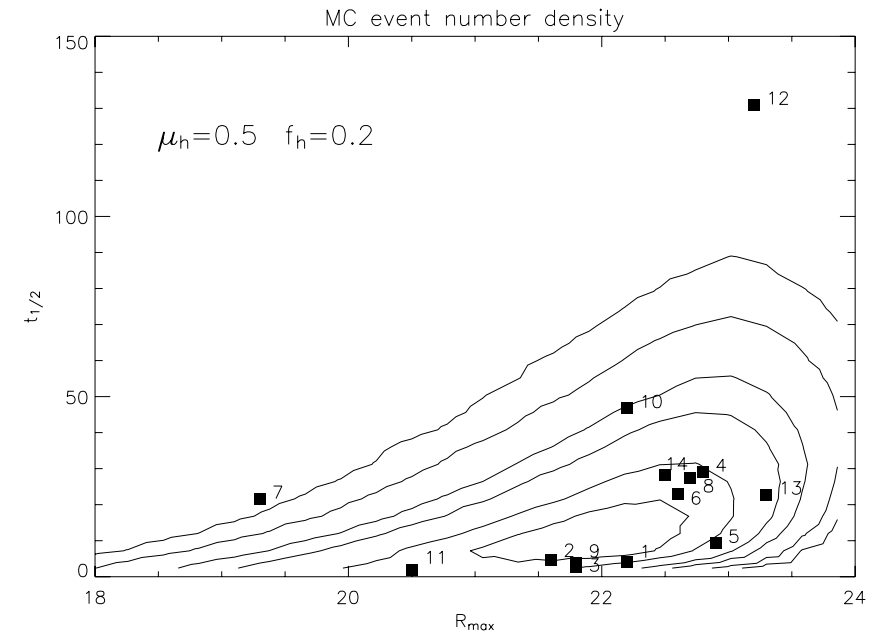

Fig. 6. $M C$ revealed event number density $\mathcal{N}_{\mathrm{ev}}^{\text {rev }}$ plot (normalized to the maximum value) as a function of $t_{1 / 2}$ and $R_{\max }$. Here we consider both self-lensing and MACHO-lensing events, averaged over all the 14 MEGA directions and we assume $\mu_{\mathrm{h}}=0.5$ and $f_{\mathrm{h}}=0.2$. From the inner to the outer part $\mathcal{N}_{\mathrm{ev}}^{\text {rev }}$ decreases with lines referring to values $0.7,0.5,0.3,0.2,0.1,0.05$.

with high signal-to-noise ratios. The fraction of MC selected events also depends on the event location through the threshold value of $R_{\max }$. This effect is further discussed in the following section.

\section{Monte Carlo results}

MC results allow to estimate the features of the revealed events which pass the adopted selection criteria. In Fig. 6, assuming a MACHO mass $\mu_{\mathrm{h}}=0.5$ and a halo MACHO fraction $f_{\mathrm{h}}=0.2$ we give the contour plot, in the $\left(t_{1 / 2}, R_{\max }\right)$ parameter space, of the event number density

$\mathcal{N}_{\mathrm{ev}}^{\mathrm{rev}}=\frac{\mathrm{d}^{2} N_{\mathrm{ev}}^{\mathrm{rev}}}{\mathrm{d} t_{1 / 2} \mathrm{~d} R_{\max }}$,

averaged on all the 14 MEGA directions, for both self-lensing and MACHO-lensing events. We also give in the same parameter space the position of the 14 observed MEGA events. $\mathcal{N}_{\mathrm{ev}}^{\text {rev }}$ is maximum in the region $t_{1 / 2} \simeq 20$ day and $R_{\max } \simeq 22$. Moreover, $\mathcal{N}_{\mathrm{ev}}^{\text {rev }}$ rapidly decreases for $R_{\max }>23.5$ (due to the lack of revealed events with low signal-to-noise ratio) and $t_{1 / 2}<5$ day (due to the peak sampling) and in the region of high amplification and long duration events (upper-left region of the figure), due to the absence of MC generated events. Actually, the last cutoff depends on the adopted MACHO mass value and shifts towards smaller $t_{1 / 2}$ values decreasing $\mu_{\mathrm{h}}$. Indeed, in Fig. 6 the crucial parameter determining the event distribution is the lens mass value and it turns out that the region where $\mathcal{N}_{\mathrm{ev}}^{\mathrm{rev}}$ is maximum scales with $\mu_{\mathrm{h}}$ in the same way as $t_{1 / 2}^{\text {median }}$ and $R_{\max }^{\text {median }}$ (see Table 4 , where we give the median values of the distributions of the MC revealed events as a function of the lens star mass $\mu_{*}$, $t_{1 / 2}$ and $R_{\max }$ ).

From Table 4 for $\mu_{\mathrm{h}}=0.5$ one can infer that the $\mathcal{N}_{\mathrm{ev}}^{\mathrm{rev}}$ plot does not vary substantially for MACHO-lensing and selflensing events occurring away from the M31 center, since the 


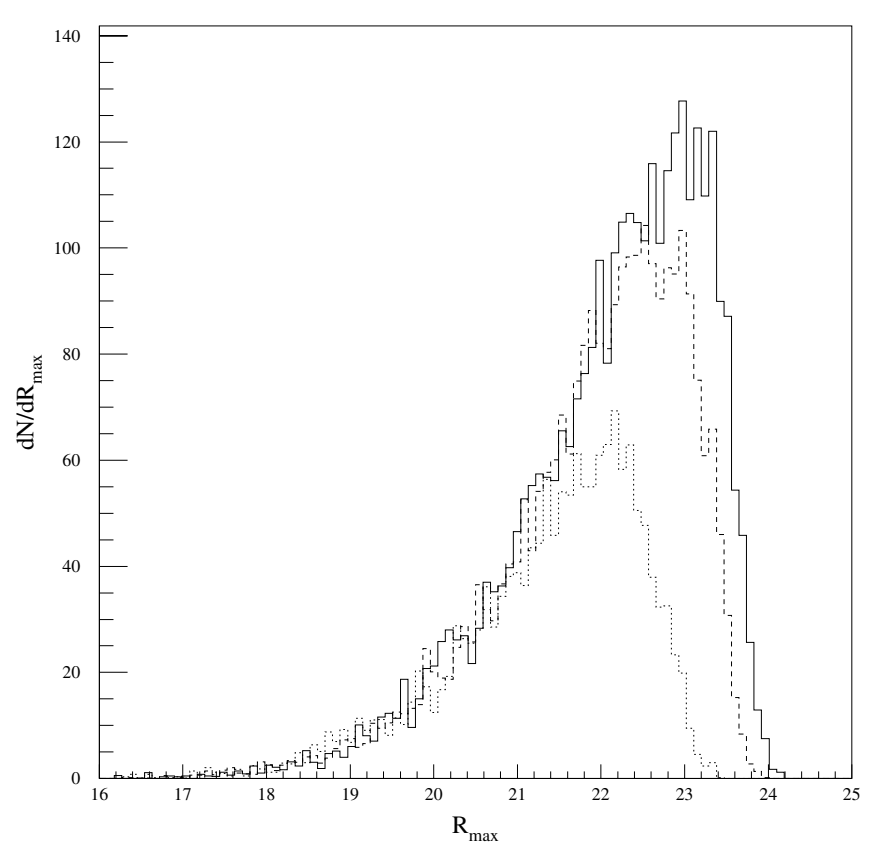

Fig. 7. The $R_{\max }$ distribution for the MC revealed events towards the MEGA 14 (solid line), 7 (dashed line) and 1 (dotted line) directions.

lens stellar mass is of the same order of the MACHO mass. Moreover, for any value of $\mu_{\mathrm{h}}$, the $\mathcal{N}_{\mathrm{ev}}^{\text {rev }}$ distribution is also weakly dependent on the selected direction towards M31. The situation is completely different for $\mu_{\mathrm{h}} \neq 0.5$, and this means that self-lensing and MACHO-lensing events lie on different regions in the $\left(t_{1 / 2}, R_{\max }\right)$ parameter space of the corresponding $\mathcal{N}_{\mathrm{ev}}^{\mathrm{rev}}$ plot.

The dependence of $t_{1 / 2}^{\text {median }}$ and $R_{\max }^{\text {median }}$ on the lens mass and the MEGA direction is also clear from Table 4 . For selflensing events, $t_{1 / 2}^{\text {median }}$ increases from the inner to the outer part of the M31 galaxy, and in this region $t_{1 / 2}^{\text {median }}$ also increases for increasing values of $\mu_{*}^{\text {median }}$, following the dependence of the Einstein radius with the lens mass ${ }^{4}$. Moreover, for MACHOlensing events with a fixed $\mu_{\mathrm{h}}$ value, $t_{1 / 2}^{\text {median }}$ is almost the same for any MEGA direction, while it increases, as expected, with the lens mass.

In Table 4 is also evident the decrease, moving towards the M31 center, of the $R_{\max }^{\operatorname{median}}$ value that follows the surface brightness variation along the field. In particular, we find a shift of about 1 mag going from the inner to the outer regions. In Fig. 7 we give the (revealed) event distributions as a function of $R_{\max }$ towards the MEGA 1, 7 and 14 directions. This clearly shows how the event location affects the fraction of the revealed events.

The event distributions as a function of $t_{1 / 2}$, for both selflensing (solid line) and MACHO-lensing (dashed line) revealed events are shown in Fig. 8, for $\mu_{\mathrm{h}}=0.5$ and for the 1 and 7 MEGA directions towards M 31 (representative of inner and outer events, respectively). Only in the case of the MEGA 1

${ }^{4}$ For self-lensing events, the lens median mass value changes with the MEGA direction, since the disk lens mass is on average higher than the bulge lens mass and the disk-to-bulge probability ratio depends on the MEGA direction.


Fig. 8. The $t_{1 / 2}$ distributions are shown for MEGA 1 and 7 events and for $\mu_{\mathrm{h}}=0.5$. In each panel, the solid curve refers to self-lensing events and the dashed curve to MACHO-lensing events.

direction the obtained distributions are markedly different for self-lensing and MACHO-lensing, since self-lensing events are shorter than MACHO-lensing events. For the MEGA 7 direction (and the other outer directions) self-lensing and MACHO lensing with the same lens mean mass have roughly the same $t_{1 / 2}$ distributions.

Our MC results can be used to estimate the lens nature and location of the 14 MEGA candidate events, by weighting the microlensing rate - giving the analytical estimates of the MACHO-to-self lensing probability ratio $\left(P_{\mathrm{h}} / P_{\mathrm{s}}\right)_{\mathrm{An}}$ shown in Table 2 - with the revealed event number density distribution and taking also into account the observed features of the MEGA events. In Table 5, assuming a halo MACHO 

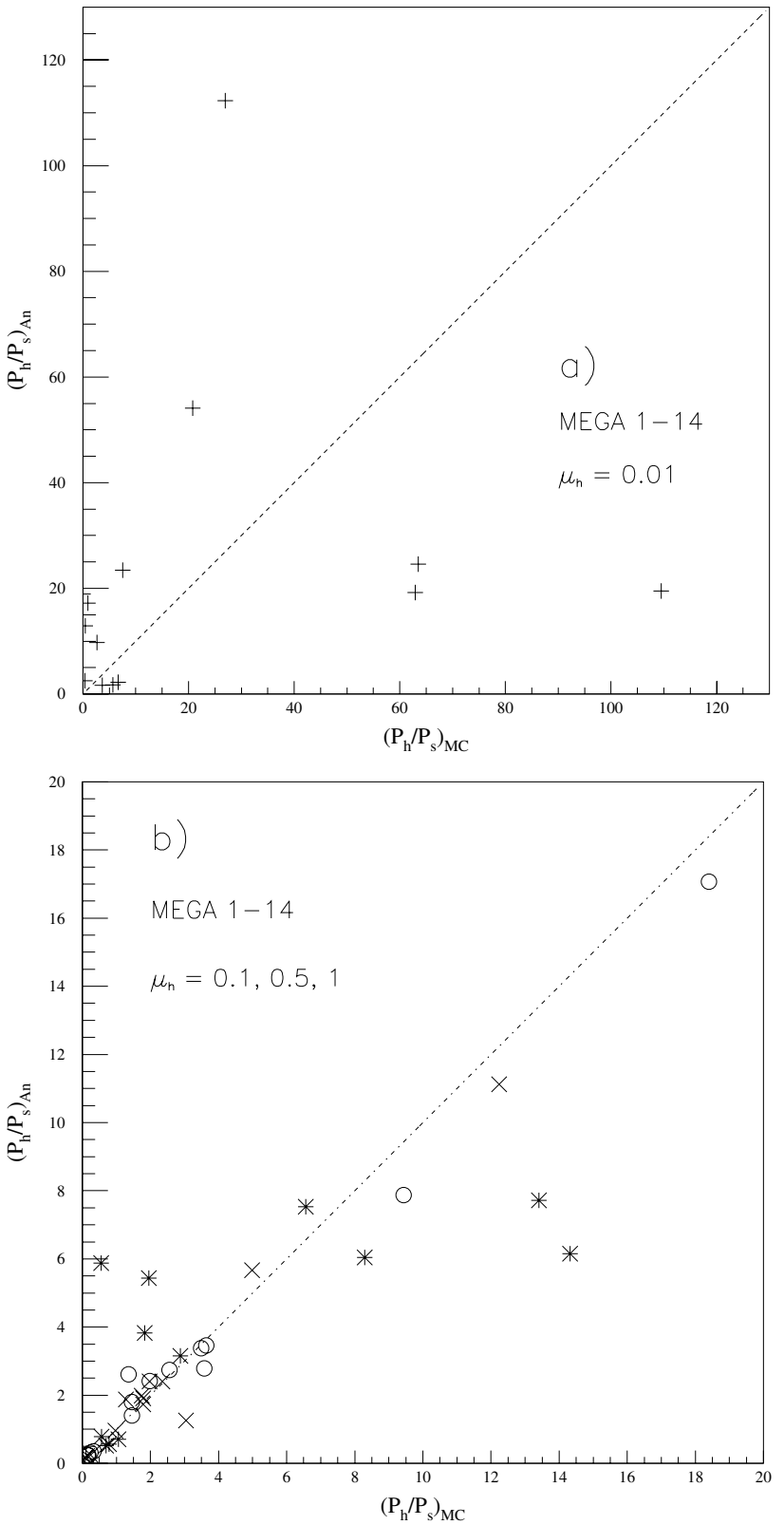

Fig. 9. Plot of $\left(P_{\mathrm{h}} / P_{\mathrm{s}}\right)_{\mathrm{An}}$ versus $\left(P_{\mathrm{h}} / P_{\mathrm{s}}\right)_{\mathrm{MC}}$ for all the 14 MEGA events. In panel a) we consider a MACHO mass $\mu_{\mathrm{h}}=0.01$ and we use the symbol plus; in panel b) we take $\mu_{\mathrm{h}}=0.1,0.5,1$ and use symbols time, circle, asterisk, respectively.

fraction $f_{\mathrm{h}}=0.2$ and different MACHO mass values, we give the MC MACHO-to-self lensing probability ratio

$\left(\frac{P_{\mathrm{h}}}{P_{\mathrm{s}}}\right)_{\mathrm{MC}}=\left(\frac{P_{\mathrm{h}}}{P_{\mathrm{s}}}\right)_{\mathrm{An}} \frac{\mathcal{N}_{\mathrm{ev} \mathrm{h}}^{\mathrm{rev}}\left(t_{1 / 2}^{\mathrm{obs}}\right)}{\mathcal{N}_{\mathrm{ev} \mathrm{s}}^{\text {rev }}\left(t_{1 / 2}^{\mathrm{obs}}\right)} \frac{\mathcal{N}_{\mathrm{ev} \mathrm{h}}^{\text {rev }}\left(R_{\mathrm{max}}^{\mathrm{obs}}\right)}{\mathcal{N}_{\mathrm{ev} \mathrm{s}}^{\text {rev }}\left(R_{\max }^{\text {obs }}\right)}$

where we indicate with $\mathcal{N}_{\mathrm{ev}}^{\mathrm{rev}}\left(t_{1 / 2}^{\text {obs }}\right)$ and $\mathcal{N}_{\mathrm{ev}}^{\mathrm{rev}}\left(R_{\max }^{\mathrm{obs}}\right)$ the number of events (either MACHO or self-lensing) with duration and magnitude at maximum, respectively, within 2 standard deviations around the observed values. Here, we remark that each $\mathcal{N}_{\text {ev }}$ distribution is normalized to the total number of revealed events. A comparison between the analytical and MC results given, respectively, in Tables 2 and 5 is presented in Figs. $9 \mathrm{a}$ and $9 \mathrm{~b}$, where we plot $\left(P_{\mathrm{h}} / P_{\mathrm{s}}\right)_{\mathrm{An}}$ versus $\left(P_{\mathrm{h}} / P_{\mathrm{s}}\right)_{\mathrm{MC}}$,
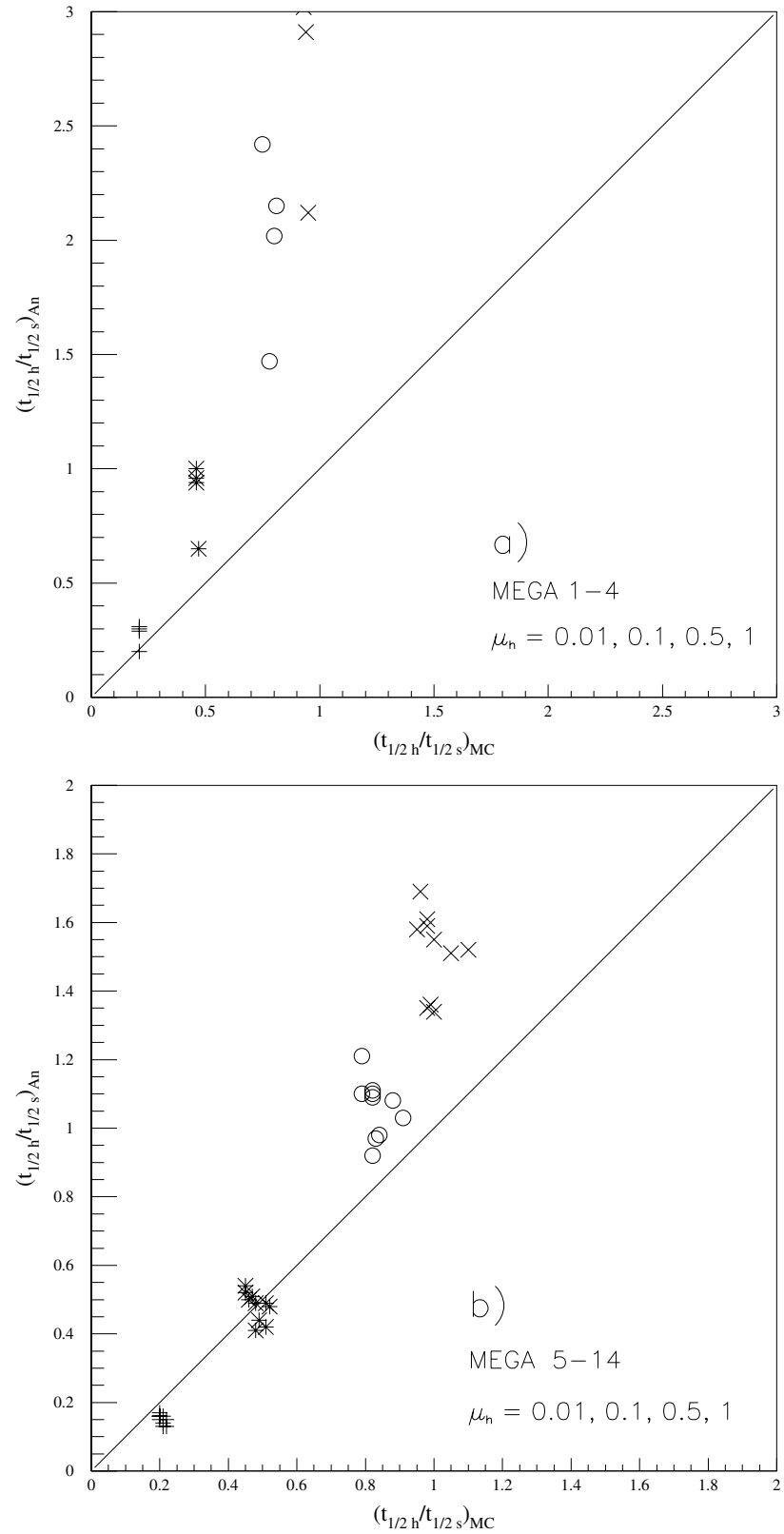

Fig. 10. Plot of $\left(t_{1 / 2 \mathrm{~h}} / t_{1 / 2 \mathrm{~s}}\right)_{\mathrm{An}}$ versus $\left(t_{1 / 2 \mathrm{~h}} / t_{1 / 1 \mathrm{~s}}\right)_{\mathrm{MC}}$, for $\mu_{\mathrm{h}}=$ $0.01,0.1,0.5,1$. The symbols are as in Fig. 9. In panel a) we consider MEGA 1-4 and in panel b) MEGA 5-14 events, respectively.

for the MACHO mass values $\mu_{\mathrm{h}}=0.01$ and $\mu_{\mathrm{h}}=0.1,0.5,1$, respectively. Only for $\mu_{\mathrm{h}}=0.5$ and for the outer events MC and analytical estimates are in good agreement, since, as we have already discussed above, MACHO-lensing and selflensing events have for this particular mass value, on average, the same features.

Also for the time scale ratios between MACHO-lensing and self-lensing events analytical and MC results give different estimates. A comparison of the analytical results in Table 3 and MC results in Table 4 is presented in Figs. 10a and $10 \mathrm{~b}$, where we show $\left(t_{1 / 2 \mathrm{~h}} / t_{1 / 2 \mathrm{~s}}\right)_{\mathrm{An}}$ versus $\left(t_{1 / 2 \mathrm{~h}} / t_{1 / 1 \mathrm{~s}}\right)_{\mathrm{MC}}$ for MEGA 1-4 and 5-14 events. The difference is particularly important for the events $1-4$. 
It emerges, therefore, clearly that the MC analysis is essential for determining the lens nature and location of microlensing events, at least if the MACHO mass differs substantially from the average self-lensing mass. From Table 5, we find that MEGA events 1-4 are most likely self-lensing events for MACHO masses greater than 0.1 solar masses, while events 5, 6, 9, 11, 13, 14 are likely MACHO-lensing. For the other MEGA directions the lens nature is more uncertain. As a final comment in Fig. 6, we note that the event 12, lying in a region of low (revealed) event number density, hardly can be considered a reliable microlensing event, unless the MACHO mass is considerably greater than $1 M_{\odot}$.

\section{Conclusions}

We have studied the main features of the expected microlensing events in pixel lensing observations towards M 31, by using both analytical estimates (from the microlensing rate) as well as results by a MC code where we reproduce the observing and instrumental conditions of the MEGA experiment.

First of all, we derive in Sect. 2 the microlensing rate, and assuming a specific mass distribution model for M 31 and the Galaxy, we calculate the MACHO-to-self lensing probability and the MACHO-to-self lensing event time scale ratios. For $\mu_{\mathrm{h}}>0.1$, we find that self-lensing dominates in the M 31 central regions. Moreover, for $\mu_{\mathrm{h}} \simeq 0.5$, towards the innermost MEGA directions, MACHOs events have duration twice as long as self-lensing events, while outer events have roughly the same duration.

We then generate a large number of MC microlensing events by choosing relevant source and lens parameters as outlined in Sect. 5. We study the observability of the MC events, by considering the capabilities of the INT Telescope, typical observing conditions and the event selection criteria adopted by the MEGA Collaboration.

MC results can be used to evaluate, for the 14 MEGA candidate events, the MACHO-to-self lensing probability and the event time scale ratios $\left(P_{\mathrm{h}} / P_{\mathrm{s}}\right)_{\mathrm{MC}}$ and $\left(t_{1 / 2 \mathrm{~h}} / t_{1 / 2} \mathrm{~s}\right)_{\mathrm{MC}}$ (given in Tables 4 and 5), by taking into account not only the analytical expectations from the microlensing rate (as already done in Tables 2 and 3 ) but also the features of the MC (revealed) events and the observed values of $t_{1 / 2}$ and $R_{\max }$. MC results and analytical expectations are compared in Figs. 9 and 10, where one can see that in determining the lens nature and location of the MEGA candidate events, the MC analysis is particularly important for $\mu_{\mathrm{h}} \neq 0.5$. Accordingly, we find that event 12, lying in a region of low event number density, hardly can be considered a reliable microlensing event, unless the MACHO mass is considerably larger than $1 M_{\odot}$. Moreover, for a MACHO mass greater than $0.1 M_{\odot}$, the innermost MEGA events $1,2,3,4$ are most likely self-lensing events, while 5, 6, 9, 11, 13, 14 are MACHO-lensing events. For the other MEGA directions the lens nature is more uncertain.
Acknowledgements. SCN is supported by the Swiss National Science Foundation.

\section{References}

Alcock, C., Akerloff, C. W., Allsman, R. A., et al. 1993, Nature, 365, 621

Alcock, C., Allsman, R. A., Alves, D. R., et al. 2000, ApJ, 542, 281

An, J. H., Evans, N. W., Kerins, E., et al. 2004, ApJ, 601, 845

Ansari, R., Auriere, M., Baillon, P., et al. 1997, A\&A, 324, 843

Aubourg, E., Bareyre, P., Brehin, S., et al. 1993, Nature, 365, 623

Baillon, P., Bouquet, A., Giraud-Heraud, Y., \& Kaplan, J. 1993, A\&A, 277,1

Baltz, E. A., Gyuk, G., \& Crotts, A. P. S. 2003, ApJ, 582, 30

Belokurov, V., An, J., Evans, N. W., et al. 2005, MNRAS, 357, 17

Binney, J., \& Tremaine, S. 1987, Galactic Dynamics (Princeton, New Jersey: Princeton University Press)

Calchi Novati, S., Iovane, G., Marino, A., et al. 2002, A\&A, 381, 845

Calchi Novati, S., Jetzer, Ph., Scarpetta, G., et al. 2003, A\&A, 405, 851

Calchi Novati, S., Paulin-Henriksson, S., Baillon, P., et al. 2005, A\&A, 443, 911

Crotts, A. P. S. 1992, ApJ, 399, L43

de Jong, J. T. A., Kuijken, K., Crotts, A. P. S., et al. 2004, A\&A, 417, 461

De Paolis, F., Ingrosso, G., Nucita, A., \& Zakharov, A. F. 2005, A\&A, 432, 501

De Rújula, A., Jetzer, Ph., \& Masso, E. 1991, MNRAS, 250, 348

Gondolo, P. 1999, ApJ, 510, L29

Gould, A., Bahcall, J. N., \& Flynn, C. 1997, ApJ, 482, 913

Griest, K. 1991, ApJ, 366, 412

Gyuk, G., \& Crotts, A. P. S. 2000, ApJ, 535, 621

Jetzer, Ph. 1994, A\&A, 286, 426

Jetzer, Ph., Milsztajn, A., \& Tisserand, P. 2004, in Proc. IAU Symp. 225, ed. Y. Mellier, \& G. Meylan (Cambridge University Press 2005), 339

Jetzer, Ph., Mancini, L., \& Scarpetta, G. 2002, A\&A, 393, 129

Joshi, Y. C., Pandey, A. K., Narasimha, D., \& Sagar, R. 2005, A\&A, 433, 787

Kent, S. M. 1989, AJ, 97, 1614

Kerins, E., Carr, B., Ewans, N. W., et al. 2001, MNRAS, 323, 13

Kerins, E., An, J., Ewans, N. W., et al. 2003, ApJ, 598, 993

Kerins, E. 2004, MNRAS, 347, 1033

Kerins, E., Darnley, M. J., Duke, J., et al. 2005

[arXiv:astro-ph/0502545]

Mamon, G. A., \& Soneira, R. M. 1982, ApJ, 255, 181

Navarro, J. F., Frenk, C. S., \& White, S. D. M. 1997, ApJ, 490, 493

Paczyński, B. 1986, ApJ, 304, 1

Paulin-Henriksson, S., Baillon, P., Bouquet, A., et al. 2003, A\&A, 405, 15

Riffeser, A., Fliri, J., Bender, R., et al. 2003, ApJ, 599, L17

Stark, A., \& Binney, J. 1994, ApJ, 426, L31

Uglesich, R. R., Crotts, A. P. S., Baltz, E. A., et al. 2004, ApJ, 588, 311 